\title{
Thermodynamic Properties of Compressed Solid Hydrogen*
}

\author{
G. A. NEECE ${ }^{\dagger}$, F. J. Rogers, AND W. G. HoOver \\ Lawrence Radiation Laboratory, University of California, Livermore, California
}

Received November 18, 1970

\begin{abstract}
The high-pressure thermodynamic properties of molecular and metallic hydrogen are calculated from the fundamental viewpoint of statistical mechanics. In the molecular solid phase the interaction between hydrogen molecules is taken to be an angle-dependent de Boer repulsion, fitted to the theoretical results of Hirschfelder, Magnasco, Mason, McWeeny, and Musso, with spherical attractions as calculated by Margenau. Thermodynamic properties for the denser metallic phase are obtained from an improved selfconsistent version of the Wigner-Huntington calculation, in which the correlations due to electronic repulsions are more closely approximated and in which the Kohn-Sham local exchange is used. By comparing the Gibbs free energies of the molecular solid and the metal, we predict a phase transition linking the two at $840 \mathrm{kbars}$, well within the reach of several experiments now being designed.
\end{abstract}

\section{TNTRODUCTION}

From the fundamental viewpoint of the theoretical physicist, hydrogen has a unique fascination: It is the simplest aton of all, with only one electron and one proton. If the physicist today, with the aid of powerful computers, is unable to calculate, rather than merely to measure experimentally the physical properties of this most elementary of all substances, it can be said that he understands very little! Hydrogen is also interesting from other points of view. Astrophysicists are interested not only in hydrogen in stars but also in planets. Saturn and Jupiter are mostly hydrogen, under pressures up to $100 \mathrm{Mbars}$ [1]. Astrophysical calculations of the structure of these planets require a hydrogen equation of state at high pressures. More practically speaking, nothing is more basic to our very existence than hydrogen. The tremendous energy generated by its fusion in the sun is the basic source of all life on earth.

The tremendous energy source provided by hydrogen fusion is already available in bydrogen bombs and is of potential catastrophic harm to us all. Soon, the energy of hydrogen will be available in usable form, an inexhaustible source of power in

* Work performed under the auspices of the U. S. Atomic Energy Commission.

+ Summer visitor. Permanent address: University of Georgia, Athens, Georgia. 
fusion reactors, allowing us to reduce our consumption of the dwindling fossil fuels [2].

For all these reasons hydrogen is studied. Despite this focus of scientific interest, estimates of its thermodynamic properties vary widely once we leave the region accessible to direct experiment. At high density, as Bernal suggested, the molecular crystal formed by compressing the gas or liquid, on further compression becomes unstable relative to a denser metallic form. Estimates of the transition pressure from the molecular solid to the metallic solid vary all the way from 0.25 to 20 megabars $[3,4]$.

Experiments are now being prepared [5] which should soon settle the question of the high-density hydrogen equation of state. Thus a theoretical estimate is particularly appropriate now, both to guide the experiments and to test the theory. Fast computers make such theoretical calculations simpler than ever before, both in determining the forces with which atoms and molecules interact, and in discovering how these forces produce thermodynamic properties.

\section{The Molecular Soltd}

\section{A. Intermolecular Potential}

The ground state energy $E_{0}$ of two interacting hydrogen molecules (with fixed nuclei) is conventionally divided into two parts, the intermolecular potential energy $\phi$ and the ground state energy of two isolated molecules, $2 E\left(H_{2}\right)$; the interaction energy is $\phi \equiv E_{0}-2 E\left(H_{2}\right)$. Several studies of $\phi$ as a function of the separation and relative orientation of the two molecules have been carried out [6-12]. The most elaborate of these valence bond calculations used a variational wavefunction built up of optimized atomic orbitals describing all neutral, singly, and doubly ionized structures [10]. The atom-atom separation within each molecule was kept fixed at $0.7496 \AA$ while the separation between the molecular centers varied from $1.5-3 \AA$. The results given in Refs. [8-12] can all be summarized by the empirical repulsive potential

$$
\phi_{\mathrm{rep}}=\sum_{i=1}^{4} \epsilon e^{-r_{i} / r_{0}},
$$

where the four terms in the sum correspond to the four pairs of atoms, one in each molecule, shown in Fig. 1. The same form (1) was used by de Boer in 1942 [6]. We have adopted the constants $\epsilon=3.2 e^{2} / a_{0}\left(\epsilon / K=1.01 \times 10^{6} \mathrm{~K}\right)$ and $r_{0}=0.300 \AA$. This choice reproduces the theoretical results well. The maximum disagreements are no greater than $10-15 \%$, about the same order of magnitude as the variation in the theoretical results from the various methods used in Refs. [8-12]. 
In the preliminary calculations designed to study the ease of rotation in the solid phase we used only the repulsive part of the hydrogen-hydrogen interaction (1). For quantitative predictions of solid hydrogen thermodynamics, the attractive forces due to the interactions of induced dipoles and quadrupoles must be included. Because these attractive forces have a smaller impact on thermodynamic quantities,

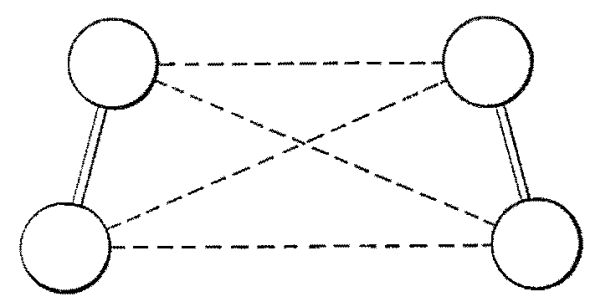

FIG. 1. Angle-dependent repulsive interations between nearest-neighbor molecules. The dashed lines represent the distances appearing in Eq. (1).

and because they depend much less strongly on orientation, we are justified in neglecting the angle dependence in the attractions. We use Margenau's results:

$$
\phi_{\text {ait }}=\left[-10.9\left(a_{0} / R\right)^{6}-116\left(a_{0} / R\right)^{8}\right] e^{2} / a_{0},
$$

where $R$ is the center-to-center separation of the two molecules [13].

\section{B. Thermodynamic Properties}

To calculate thermodynamic properties with our interaction potential $\phi=$ $\phi_{\text {rep }}+\phi_{\text {att }}$ we need to know the high-pressure crystal structure of hydrogen. Although the high-pressure structure has not yet been determined experimentally, the existence of a low-pressure cubic form of molecular hydrogen has been established [14]. Because other structures are less symmetric and seem no more likely at high pressure we use the simplest possible cubic crystal structure, the so-called " $\alpha$-Nitrogen" structure shown in Fig. 2. It seems likely that the solidphase properties are insensitive to the assumed structure.

We first carried out exploratory classical computer simulations of small (32 molecules) periodic crystals of hydrogen using the Monte Carlo method described by Wood [15]. In applying the Monte Carlo method to hydrogen it proved convenient to select and move an individual atom in proceeding from one configuration to the next. Random increments in the polar and azimuthal angles describing the orientation of the atom's molecule were selected and the atom was moved so as to keep the atom-atom separation in that molecule constant. The maximum angular jump allowed was varied with density and temperature and the 
decision as to whether or not a trial move is accepted was as usual based on comparing $e^{-\delta \phi i k T}$ with a random number between 0 and 1 .

Expressions for the energy and pressure can be derived by differentiating the canonical partition function $Z$ for $N$ bydrogen molecules:

$$
\begin{aligned}
& \frac{E}{k T}=\left(\frac{\partial \ln Z}{\partial \ln T}\right)_{V}=\frac{5 N}{2}+\left\langle\frac{\Sigma \phi}{k T}\right\rangle, \\
& \frac{P V}{k T}=\left(\frac{\partial \ln Z}{\partial \ln V}\right)_{T}=N-\left\langle\Sigma \frac{R \phi_{\mathrm{stt}}^{\prime}}{3 k T}\right\rangle-\left\langle\Sigma \frac{\mathbf{R} \cdot \mathbf{r} \phi_{\text {rep }}^{\prime}}{3 r k T}\right\rangle .
\end{aligned}
$$

The first two sums in (3) range over all pairs of molecules. The last sum is taken over all pairs of atoms in nearest-neighbor molecules. The dot product in this repulsive term arises because volume differentiation corresponds formally to separating the centers of two interacting molecules. The change in the relative separation of a pair at $r$ is proportional to the dot product $\mathbf{r} \cdot \mathbf{R}$.

Both the exploratory Monte Carlo calculations and more extensive "cell-model" calculations in which only a single particle was allowed to move, the rest being kept fixed in their perfect-lattice orientation (the orientations shown in Fig. 2),

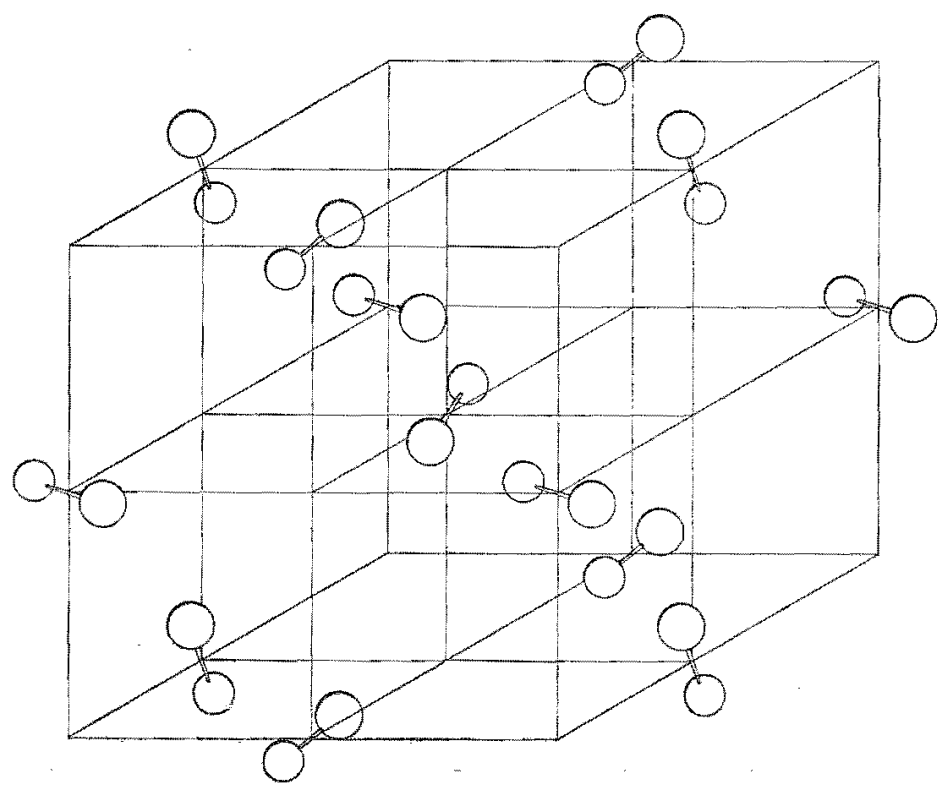

FIG. 2. Assumed crystal structure of the molecular solid, the so-called " $\alpha$-nitrogen" structure. There are four molecules in the unit cell. The molecular axes lie parallel to the body diagonals of a cube. 
indicated that the quasiharmonic approximation of lattice dynamics would furnish a semiquantitative description of the energy and pressure between 3-7 cc/mole $H_{2}$ and for temperatures up to several hundred degrees. In this range the classical Monte Carlo energy relative to a static lattice was quite close to the ideal harmonic value of $5 N k T$. This means that under these conditions the molecular rotational quantum number relevant at low densities is no longer a useful description of the molecular state; the "rotations" should instead be viewed as anharmonic vibrations.

The simplest solid-phase harmonic model is the Einstein model in which a single particle vibrates in the field of its fixed neighbors. The vibrations include three degenerate "translational" center-of-mass-motion frequencies and two degenerate "rotational" frequencies. These frequencies are listed in Table I. The barrier to rotation of a molecule (the minimum increase in potential energy necessary to interchange the two atoms in a molecule) is shown in Fig. 3. The Monte Carlo work indicates that cooperative motion reduces the effective barrier to rotation.

\section{TABLE I}

Einstein temperatures, energies, and pressures for solid molecular hydrogen. Volume is expressed in cc per mole of $H_{2} . \theta_{\text {trans }}$ and $\theta_{\text {rot }}$ are the characteristic temperatures, $h v / k$, in Kelvins, for the "translational" and "rotational" vibrations of an Einstein molecule. The energies (kbarcc/mole $\mathrm{H}_{2}$ ) and pressures (kbars) are tabulated both for a static lattice and for a lattice with zeropoint energy incorporating both the translational and rotational zeropoint energies.

\begin{tabular}{crrrrrr}
\hline Volume & $\theta_{\mathbf{v} 1 \mathrm{~b}}$ & $\theta_{\text {rot }}$ & $E_{\text {Btatic }}$ & $P_{\text {static }}$ & $E_{\text {with zp }}$ & $P_{\text {with } 2 p}$ \\
\hline 4 & 1474 & 1257 & 1544 & 900 & 1824 & 971 \\
5 & 1205 & 957 & 892 & 465 & 1116 & 513 \\
6 & 990 & 753 & 540 & 260 & 722 & 295 \\
7 & 821 & 608 & 338 & 154 & 488 & 181 \\
8 & 688 & 501 & 216 & 95 & 341 & 117 \\
9 & 583 & 419 & 140 & 61 & 245 & 78 \\
10 & 498 & 355 & 91 & 40 & 181 & 54 \\
\hline
\end{tabular}

Because the Debye model, with a quadratic frequency distribution $g(v) \alpha \nu^{2}$, is a better approximation to reality than is the Einstein model, we have used the Debye theory to calculate the contribution of the zero-point energy to the thermodynamic properties. As Rice has stressed [16], the best way to correlate the Einstein and Debye models is to equate the second moments of the two frequency distributions (as is required for consistency at high temperature):

$$
\frac{3}{5} v_{\text {Debye }}^{2}=\left\langle v^{2}\right\rangle=\frac{1}{5}\left(3 v_{\text {trans }}^{2}+2 v_{\text {rot }}^{2}\right)
$$

Our numerical results for the static-lattice energy and for the energy with the 
Debye zero-point energy (9/8)(5/3) $N h \nu_{\text {Debye }}$ added can be closely approximated by the following simple functions of the molar volume $V\left(\mathrm{cc} /\right.$ mole $\left.H_{2}\right)$ :

$$
\begin{gathered}
E_{\text {statlc }}=1810000 e^{-4,2 V^{1 / 3}}-12140 V^{-2}, \\
E_{\text {with zp }}=1129000 e^{-4.01 V^{1 / 3}}-1885 V^{-2}, \\
\theta_{\text {Debye }}=83730 e^{-2.24 V^{1 / 3}}-9525 V^{-2}
\end{gathered}
$$

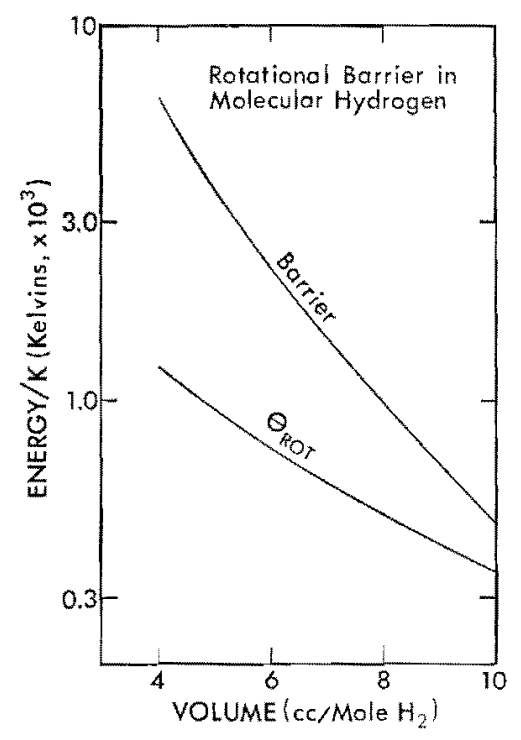

FIgURE 3

In (5) the energies are in $\mathrm{kbarcc} /$ mole $H_{2}$ and $\theta_{\text {Debye }} \equiv h \nu_{\text {Debye }} / k$ is in kelvins. The pressure-volume equation of state from (5) is shown in Fig. 4 for comparison with other theoretical estimates. Numerical values appear in Table I. Our pressure lies between that of Abrikosov [17] (who used a hexagonal crystal structure and completely ignored the contribution of attractive terms to the pressure) and Trubitsyn [18], who fitted the form (5) to Stewart's low-pressure data. Notice that our pressure agrees much better with deMarcus' extrapolation [1] of the same experimental data. We expect our results (5) to apply at volumes less than 7 or 8 $\mathrm{cc} /$ mole $H_{2}$. For lower densitics our repulsive potential overestimates the intermolecular repulsion. This is the reason why our energies and pressures near $10 \mathrm{cc}$ are much higher than the experimental ones. 


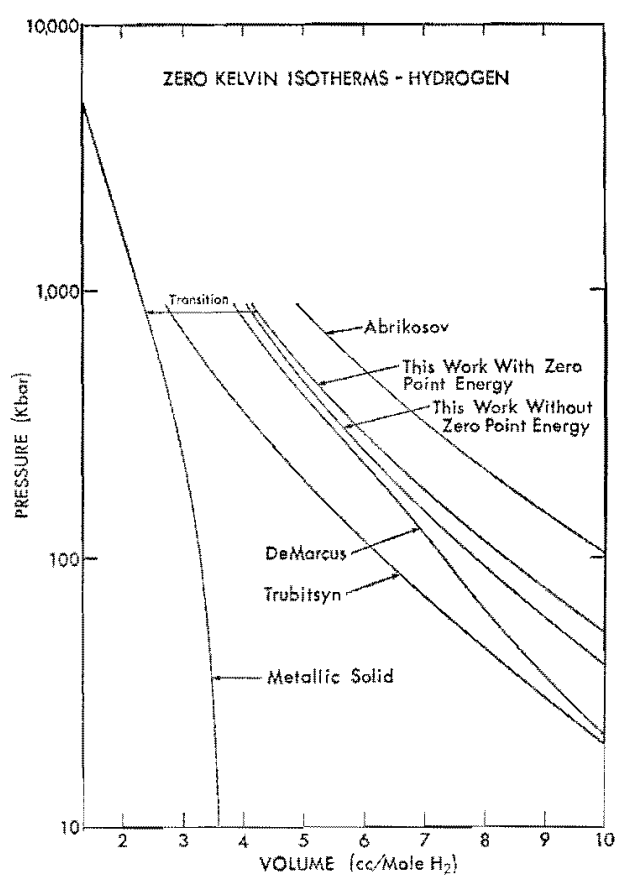

FIGURE 4

\section{The Metallic Solid}

In the alkali metals the thermodynamic properties are largely determined by the $s$-band electrons. Hydrogen which has no core electrons to perturb its $1 s$ properties should be the simplest of the alkalis. It differs from the heavier alkalis in two important ways: (1) Because there is no core of screening electrons the electrons are more tightly bound to the nuclei, giving a nonuniform charge density; (2) The small mass of the nucleus makes the zero-point energy contribution relatively more important than in the heavier alkalis.

Just as in the molecular case the high-pressure crystal structure of metallic hydrogen is not known. By analogy with the other alkalis we suppose it to be body-centered cubic. Because Carr [19] has shown that energy differences between the body-centered cubic and face-centered structures are of order 100 calories per mole we expect that thermodynamic properties are essentially independent of the metal's crystal structure.

Many earlier calculations of metallic hydrogen thermodynamics have been carried out, $[1,3,17-21]$ but without supporting calculations for the other alkalis 
which can be compared with experiment. For hydrogen we will use a revised and self-consistent Wigner-Seitz procedure, applying the same method to both lithium and sodium as well, for which experimental data are available. The good agreement in these more complicated cases justifies applying the same approach to hydrogen.

To calculate the ground-state (zero kelvino) energy of the metal, we solve the Schroedinger equation for the wavefunctions $\psi_{k}$ which minimize the HartreeFock energy [22] $E_{\mathrm{HF}}$ :

$$
\begin{aligned}
& E_{\mathrm{HF}}=2 \sum_{k} \int \psi_{h} *\left(\frac{-\hbar^{2}}{2 m} \nabla^{2}+V_{\mathrm{core}}(r)\right) \psi_{t} d \mathrm{r} \\
& +2 \sum_{k} \sum_{k^{\prime}} e^{2} \iint \frac{\psi_{k}^{2}\left(r_{1}\right) \psi_{k^{2}}^{2}\left(r_{2}\right)}{r_{12}} d \mathbf{r}_{1} d \mathbf{r}_{2}
\end{aligned}
$$

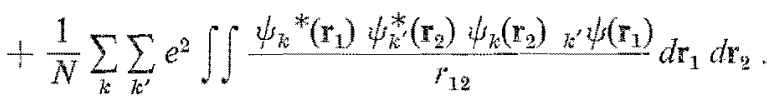

where the $\psi_{k}$ satisfy

$$
\left(\frac{-\hbar^{2} \nabla^{2}}{2 m}+V\right) \psi_{k}=E_{k} \psi_{k}
$$

and

$$
V=V_{\text {core }}+V_{\text {electrostatic }}+V_{\text {exchange and correlation }}
$$

The sums range over half a Brillouin zone and the integrals are carried out in spheres. The electrostatic and core potentials represent the interaction of an electron with the fixed nuclei and with the uncorrelated charge distributions due to all the other electrons. The exchange and correlation potential is the local effective potential of Kohn and Sham [23] in which the local charge density is assumed to contribute to the exchange and correlation energy present in an ideal electron gas at the same density. We are in effect minimizing the energy of a slightly more general equation than $\mathrm{Eq}$. (6). The free electron correlation energy interpolation formula given by Carr and Maradudin [24] is incorporated in this calculation.

One further approximation is made in the self-consistent solution. The expansion of the electronic energies in powers of $k$ is truncated after the quadratic term. It has been shown [25] that terms to this order are nearly structure independent but that the quartic term depends on the crystal structure. The errors incurred by ignoring it should be on the order of ten calories per mole, even less than those due to replacing the Wigner-Seitz cell integrals by integrals in spheres. We use the procedure of Bardeen [26] to evaluate the quadratic term. It involves a single numerical integration of the Schroedinger equation for a $p$-state out to the cell boundary using the eigenvalue obtained for the lowest valence $s$-state. The 
quadratic energy term is given in terms of values of the $s$ - and $p$-state wavefunctions on the surface of the sphere according to

$$
E_{2}=\left[\left.\frac{4 \pi}{3} r_{s}^{3} u_{0}^{2}\left(r_{s}\right)\left(\frac{r}{p} \frac{d p}{d r}-1\right)\right|_{r=r_{s}}\right] k^{2}=\alpha k^{2},
$$

where $u_{0}$ is the lowest $s$-state wavefunction and $p$ is the function obtained by the indicated $p$-state integration. To this order of approximation

$$
\psi_{k}=\left[u_{0}+i \mathbf{k} \cdot \mathbf{x}\left(\frac{p(r)}{r^{2}}-u_{0}(r)\right)\right] e^{i \mathbf{k} \cdot \mathbf{x}},
$$

where $p(r)$ is normalized such that $p(r) / r^{2}-u_{0}(r)=0$ at $r=r_{s}$.

The one other important contribution to the energy of hydrogen is the zero-point energy. Other terms such as the Van der Waals interaction, the exchange interaction between ion cores, the volume dependence of core states, and relativistic effects can be of some significance for the other alkali metals, particularly the heavier ones. In the harmonic approximation the zero-point energy is given by

$$
E_{\mathrm{zp}}=(9 / 8) N k \theta_{0},
$$

where $\theta_{0}$ is the zero-temperature Debye temperature $h v_{\text {Debye }} / k . \theta_{0}$ can be calculated from the elastic constants. Fuchs [27] has shown that the shear moduli of alkali metals are adequately described by the electrostatic contribution alone. His result is

$$
\begin{aligned}
C_{44} & =\frac{12.9 Z_{\text {eff }}^{2}}{r_{s}{ }^{4}}, \\
\left(C_{11}-C_{12}\right) / 2 & =\frac{1.74 Z_{\text {eff }}^{2}}{r_{s}{ }^{4}} .
\end{aligned}
$$

The bulk modulus at zero temperature is given by

$$
B_{0}=-V\left(\frac{d p}{d V}\right)_{0}=\left(C_{11}+2 C_{12}\right) / 3 .
$$

With these elastic constants we obtain the Debye temperature from de Launay's tables [28]. This procedure works very well for lithium and sodium and should work well for hydrogen. As an independent check we develop an interpolation formula based on the Debye $\theta_{0}$ for an isotropic continuum. For an isotropic material

$$
\theta_{0}=\frac{f(\sigma) V^{2 / 3}}{M^{1 / 2}}\left(\frac{d p}{d V}\right)_{0}^{1 / 2},
$$


where $\sigma$ is Poisson's ratio and $M$ is the atomic mass. We use values of Poisson's ratio calculated by Kopyshev [29] from an isotropic Thomas-Fermi model and require the resultant formula to fit the $\theta_{0}$ of sodium. In this approximation

$$
f(\sigma)=2700\left(0.4438 X^{1 / 8}-0.1555 X^{1 / 3}+0.0178 X^{1 / 2}\right),
$$

where $X=Z^{2} V$, pressure is in Mbars and volume in $\mathrm{cm}^{3}$ per gram. This interpolation formula reproduces experiment within about $10 \%$ for the alkali metals. The disagreement between the two methods of obtaining $\theta_{0}$ is fairly large for hydrogen both in magnitude and volume dependence. This is shown in Fig. 5. The Kopyshev $\theta_{0}$ is also plotted. It is rather similar to the $\theta_{0}$ obtained from the elastic constants.

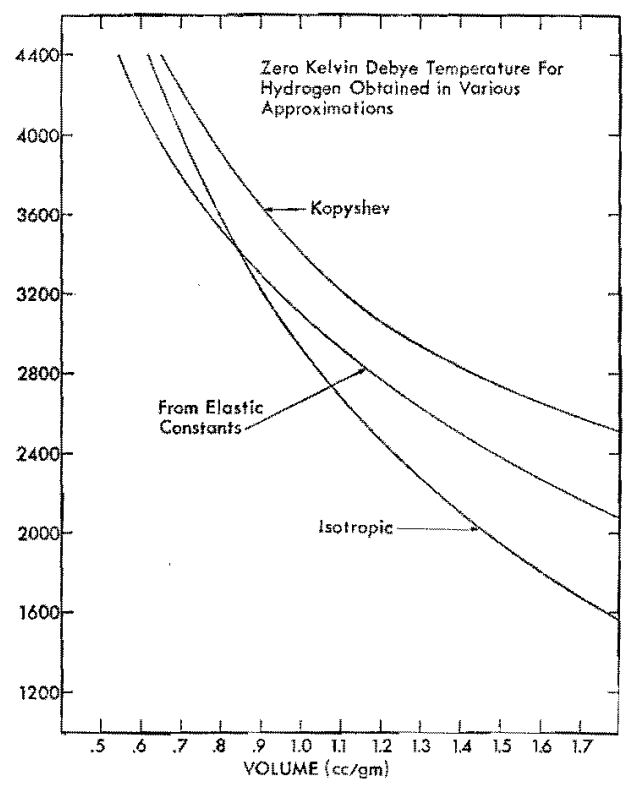

Figure 5

Finally, putting all these terms together we get for the total energy

$$
\begin{aligned}
E= & E_{0}+\frac{2.21 \alpha}{r_{s}^{2}}+\frac{1}{2} \int_{0}^{r_{s}} V_{\mathrm{es}}(r) \rho(r) d \mathbf{r}+\int_{0}^{r_{s}} \rho(r) \epsilon_{\mathrm{xc}}(\rho(r)) d \mathbf{r} \\
& +E_{\mathrm{zp}}-\frac{0.004 Z_{\mathrm{eff}}^{2}}{r_{s}^{2}},
\end{aligned}
$$


where $E_{0}$ is the ground-state electron energy, $V_{\text {es }}$ is the electrostatic potential, $\epsilon_{\mathrm{xc}}=$ free electron exchange energy + free electron correlation energy,

$$
\epsilon_{\mathbf{x c}}=-\frac{0.916}{r_{s}}-0.1303+0.0495 \ln r_{s}-0.00012 r_{s}^{2}
$$

and the last term corrects the electrostatic energy of a bcc lattice for crystal structure. We find that the iterative process to find a self-consistent potential converges in 2-3 iterations. Relatively small changes result from this iterative procedure in the energy obtained on the first iteration for hydrogen and sodium, but the differences for lithium are large.

The zero temperature thermodynamic properties for metallic hydrogen, lithium, and sodium calculated as just described appear in Table II and in Figs. 4, 6-8.

TABLE II

Zero Kelvin Thermodynamic Properties of Sodium, Lithium, and Hydrogen

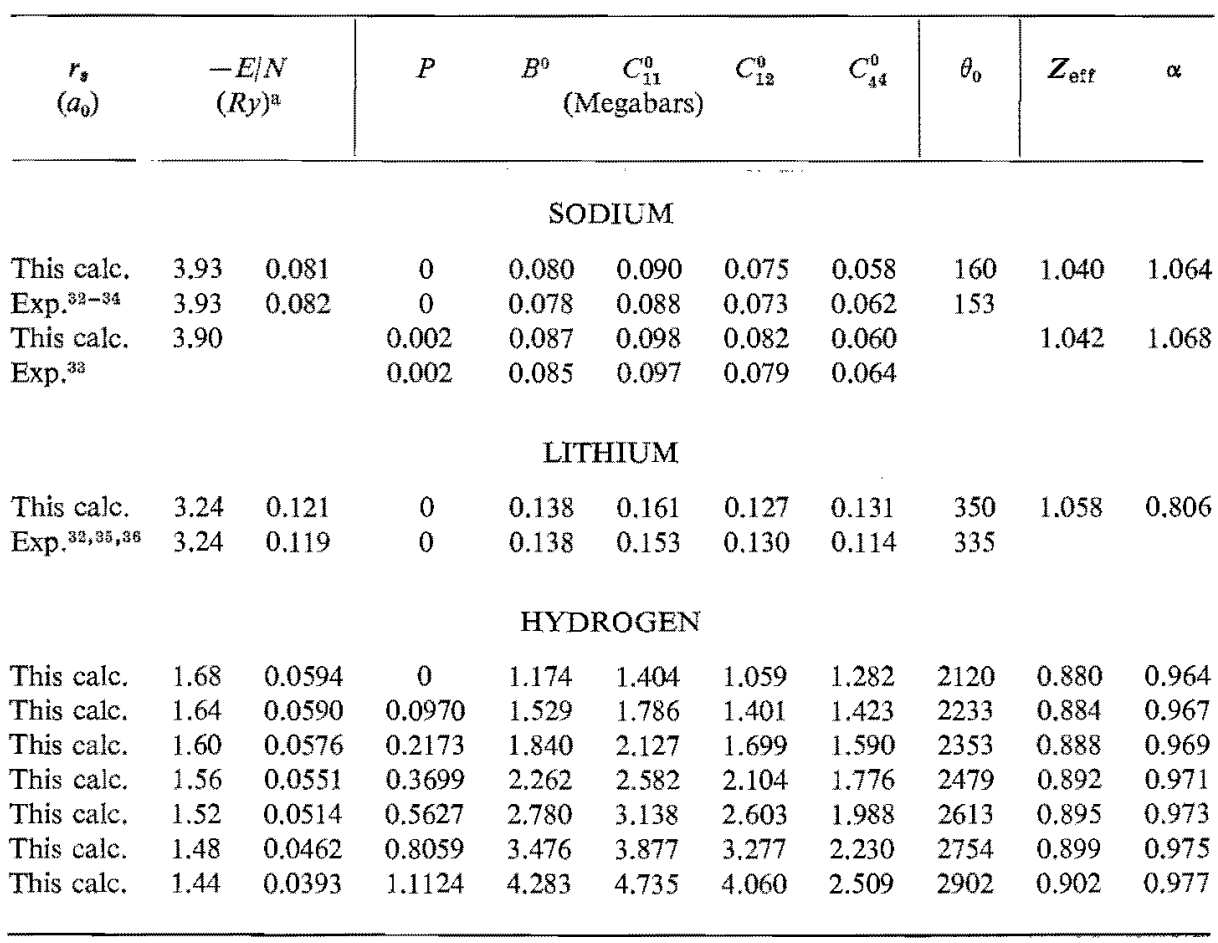

a $E$ is energy/atom relative to isolated atoms. 


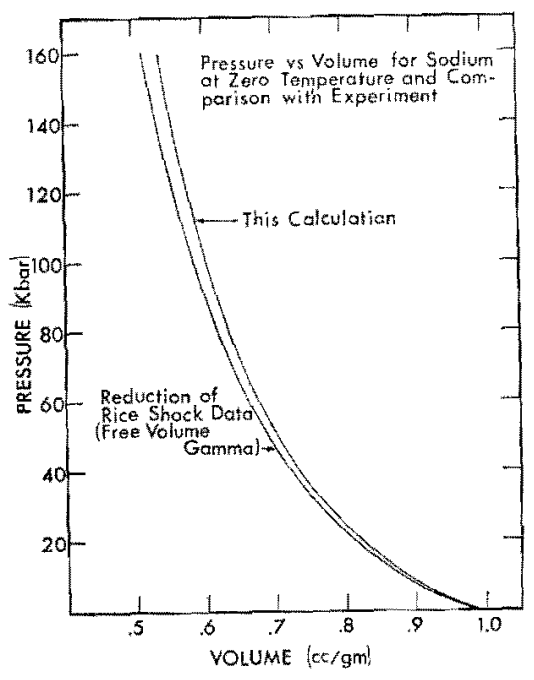

Figure 6

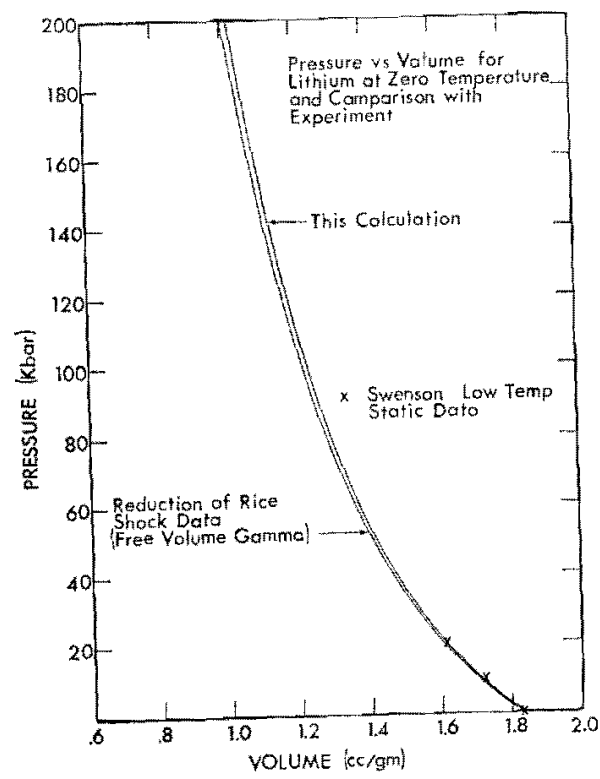

FIGURE 7 
The hydrogen results use the $\theta_{0}$ obtained from elastic constants. The core potential used for lithium was that due to Seitz [30] and that used for sodium was the one given by Wigner and Seitz [31]. The agreement with the experimental data on the two more complicated metals is very good. The slightly poorer agreement with experiment at high pressure for sodium may be due to the neglect of the aforementioned terms or the less precise core potential. There is also some uncertainty in the shock experiment and the procedure used to reduce it to zero kelvins.

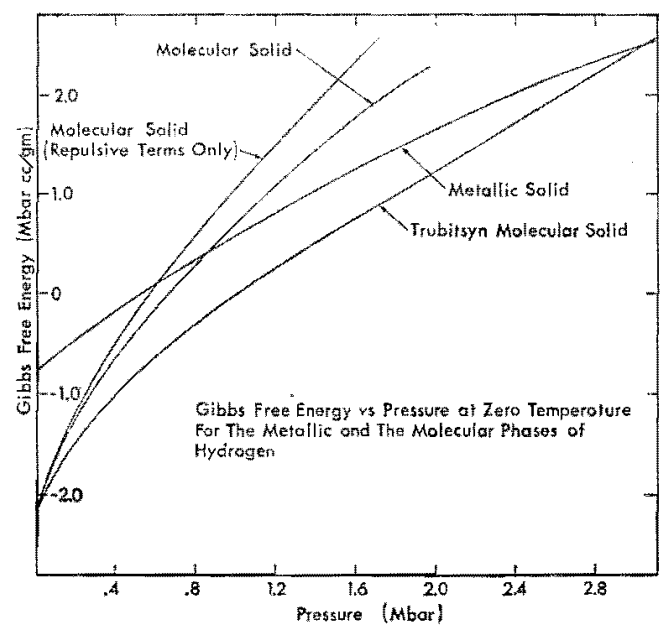

FYGLRE 8

For the hydrogen static lattice we get a cohesive energy of $0.073 \mathrm{Ry} /$ atom which is significantly larger than Wigner and Huntington's value of 0.050 Ry and Carr's value of $0.058 \mathrm{Ry}$ but less than Calais' value of $0.096 \mathrm{Ry}$. The zero-temperature $P$, $E$, and $\theta_{0}$ for pressures less than 5 mbars and with $\theta_{0}$ obtained from the elastic constants are summarized by:

$$
\begin{array}{ll}
E=E_{\text {cohesive }}-3.3214+2.1134 \ln V+\frac{3.7428}{V}, & \text { in Mbars cc/mole H } \\
P=-\frac{2.1134}{V}+\frac{3.7428}{V^{2}}, & \text { in Mbars } \\
\theta_{0}=124.7+\frac{4466.9}{V}-\frac{1858.2}{V^{2}}+\frac{372.2}{V^{3}}, & \text { in kelvins, }
\end{array}
$$

where $K$ is the volume (cc/mole H).

At temperatures above zero we use the Debye theory to estimate the contribution of the nuclear motion to thermodynamics. Because the Fermi energy is of the 
order of a few electron volts we ignore the contribution of electronic excitations. The thermal contribution to the pressure is

$$
P_{\mathrm{TH}}=\frac{\gamma_{0}\left(E-E_{0}\right)}{V}
$$

where

$$
\gamma_{0}=\frac{-d \ln \theta_{0}}{d \ln V}
$$

\section{The Transmion to a Metallic Solid}

There has been considerable theoretical interest, since the 1930's, in the possibility that molecular hydrogen becomes unstable relative to the metallic solid at high enough pressure. More recently, as experimenters learn to produce higher and higher pressures, there has been increasing interest in actually making the metal. Two approaches are being tried out: huge static presses in Russia and at Cornell; magnetic implosion at Livermore. [5]

Exactly how high a pressure is needed to produce the metal is of critical importance in designing experiments. This is because the different methods have different limits to the pressures they can reach. For this reason it is worthwile to try to set realistic limits on where such a transition is likely to occur. Past estimates have ranged from $0.25-20 \mathrm{Mbars}[3,4]$, the lower pressure being relatively easy to obtain and the higher one being out of reach at present. Most estimates are in the vicinity of 2 Mbars.

In general, one can say that the previous estimates are based on fairly similar equations of state for the metal, but wildly varying and questionable equations of state for the molecular phase. This uncertainty in the molecular phase properties was avoided by Alder [4] who used the relative interatomic distances for the other alkali metals in the gas phase and in the metal to predict this ratio for hydrogen. The extrapolation is made difficult because the alkali metals other than hydrogen are already stable relative to the molecular solid at zero pressure-calculations for the metastable diatomic solids would probably show a transition pressure becoming increasingly negative with increasing atomic number.

In Fig. 8 the zero kelvin Gibbs free energy is shown as a function of pressure for the metallic and molecular solids. The larger low-pressure compressibility of the molecular form is responsible for the characteristic difference between the metal and molecular phase at low pressure. At higher pressures the two curves differ less. Trubitsyn's predicted free energy is based on a much softer molecular equation of state than ours, leading to much smaller volumes (given by the slope in the plot) 
at high pressures and to a much higher estimate $(3.1 \mathrm{Mbars})$ for the transition pressure. Our own estimate is 840 kbars. The volume change at the transition is quite large, from $2.09 \mathrm{cc} / \mathrm{gm}$ in the molecular solid to $1.20 \mathrm{cc} / \mathrm{gm}$ in the metal. The corresponding transition for deuterium would be at a lower pressure (by about $50 \mathrm{kbar}$ ) because of the relative lowering of the metal free energy by the changed zero-point energy. Zero-point motion is more important in the metal than in the molecular solid because the metal's density is greater.

The Gibbs free energy obtained by using the repulsive part of the $\mathrm{H}_{2}-\mathrm{H}_{2}$ interaction is also plotted to demonstrate the smallness of the effect of the attractive forces. The zero-kelvin free energy for the metal is essentially the same whether the zero-point energy is calculated from the elastic constants or from the istropic approximation, Eq. (12). However, there is a significant difference at higher temperatures between the predictions of the two methods.

At finite temperatures the transition pressure changes slightly with temperature. If we continue to apply the Debye model to the molecular phase then the two versions of the Debye $\theta$ for the metal lead to estimates of $1010 \mathrm{kbar}$ (elastic constants) and $860 \mathrm{kbar}$ (isotropic) for the transition at 3000 kelvins.

In view of the good agreement with experiment found for lithium and sodium we feel justified in expecting errors no greater than $0.1 \mathrm{Mbar} \mathrm{cc} / \mathrm{gm}$ in the metal's free energy. This corresponds to an uncertainty no greater than $200 \mathrm{kbar}$ in the transition pressure. There is no reliable way to check the molecular hydrogen calculations against experiment since the experimental data only extend to $20 \mathrm{kbar}$ and our calculations are not expected to be accurate at such a low pressure. If the uncertainty in the molecular phase were similar to that found for the metal, the combined uncertainty in the transition pressure would be $400 \mathrm{kbar}$.

\section{ACKNOWLEDGMENTS}

The authors would like to acknowledge several valuable discussions with Richard Grover and David Brust concerning metal equations of state. We also would like to express our appreciation to Roger McLain for drawing the outstanding figures that appear in this paper.

\section{REFERENCES}

1. W. C. DeMARcus, Astron. J. 63 (1958), 2.

2. T. K. Fowler AND R. F. POST, Sci. Amer. 215 (1966), 21.

3. E. Wigner and H. B. Huntington, J. Chem. Phys. 3 (1935), 764. "One calculates easily, that even under the assumption of the most advantageous compressibility at high pressures, the pressure necessary for the transformation is $250000 \mathrm{atms.} . . . "$

4. B. J. ALder, in "Progress in Very High Pressure Research," (F. P. Bundy, W. R. Hibbard, and H. M. Skrong, Eds.), Wiley, New York, 1961. 
5. E. Gross, Science News 97 (1970), 623. Summarizes the experimental efforts at Livermore and Cornell. There appear to be no published descriptions of the Russian high-pressure ( 2 Mbar) press mentioned by Gross. A smaller three-stage apparatus is described by L. F. Vereshichagin, A. A. Semerchan, N. N. Kuzin, and Yu. A. Sadkov in Sov. Phys. Dokl. $13(1969), 1165$.

6. J. DE BoER, Physica 9 (1942), 363.

7. A. A. Evett and H. Margenau, Phys. Rev. 90 (1953), 121.

8. E. A. MAsON ANd J. O. Hrrschfelder, J. Chem. Phys. 26 (1957), 756.

9. V. Magnasco and G. F. Masso, J, Chem. Phys. 46 (1967), 4015.

10. V. Magnasco and G. F. Musso, J. Chem. Phys. 47 (1967), 1723.

11. V. Magnasco, G. F. Musso, And R, MoWeeny, f. Chem. Phys. 47 (1967), 4617.

12. V. Magnasco And G. F. Musso, $J$. Chem. Phys. 47 (1967), 4629.

13. H. Margenau, Phys. Rev. 64 (1943), 131.

14. Lothar Mayer, Advan. Chem. Phys. 16 (1969), 343.

15. W. W. WoOD in "Physics of Simple Liquids" (H. N. V. Temperley, J. S. Rowlinson, and G. S. Rushbrooke, Eds.) Chap. 5, Wiley, New York, 1968.

16. O. K. RICE, "Statistical Mechanics, Thermodynamics, and Kinetics," Section 9-3, Freeman, San Francisco, 1967.

17. A. A. Abrikosov, Astron. Zh. 31 (1954), 112.

18. V. P. Trubitsun, Sov. Phys. Solid State 7 (1966), $2708 ; 8$ (1966), 688.

19. W. J. Carr, Phys. Rev. 128 (1962), 120.

20. J. L. Calais, Arkiv Fysik 29 (1965), 255.

21. T. SCHNEIDER, Helv. Phys. Acta 42 (1969), 957.

22. F. SEITZ, "Modern Theory of Solids," MeGraw-Hill, New York, 1940.

23. W. Korn AND L. J. Sham, Phys. Rev, 140 (1965), A 1133.

24. W. J. Carr and A. A. Maradudin, Phys. Rev. 133 (1963), A371.

25. H. Brooks, Nuovo Cimento 7 (1958), 207; A. Bienenstock Ano H. Brooks, Phys. Rev. 136 (1964), A787.

26. J. BARDEEN, $J$, Chem. Phys. $6(1938), 367$.

27. K. Fuchs, Proc. Roy. Soc. A153 (1936), 622; A157 (1936), 444.

28. G. A. Alers, Use of sound velocity measurements, in "Physical Acoustics III," (Warren P. Mason, Ed.), Part B, Academic Press, New York, 1965.

29. V. P. Kopyshev, Sov. Phys. Dokl. 10 (1965), 338.

30. F. SEITZ, Phys. Rev. 47 (1935), 400.

31. E. Wigner And F. Seitz, Phys. Rev. 43 (1933), 804.

32. K. A. Gschnetoner, JR., Solid State Plys. 16 (1964), 344.

33. P. S. Ho AND A. L. Ruoff, J. Phys. Chen. Solids 29 (1968), 2101.

34. C. A. Swenson, Phys. Rev. 99 (1955), 423; R. I. Beecroft and C. A. Swenson, J. Phys. Chem. Solids 18 (1961), 327.

35. H. C. Nash and C. S. SMITH, J. Phys, Chem. Solids 9 (1959), 113.

36. C. A. Swenson, J. Phys. Chem. Solids 27 (1966), 33. 